\title{
LARGE HELICON APPARATUS FOR PARAMETRIC MICROWAVE COUPLING EXPERIMENTS IN A MAGNETISED PLASMA
}

K. Wilson, L. Selman, C.G. Whyte, P. MacInnes A.R. Young, A.D.R. Phelps, A.W. Cross, L. Zhang, B. Eliasson, D.C. Speirs, C.W. Robertson, and K. Ronald Department of Physics, SUPA, University of Strathclyde, Glasgow, G4 ONG, Scotland, UK

R.A. Cairns, School of Mathematics and Statistics, University of St Andrews, KY16 9SS, Scotland, UK

R. Bingham and R. Bamford STFC Rutherford Appleton Laboratory, Harwell, Oxford, Didcot, OX11 0QX, England, UK

M.E. Koepke, Department of Physics, West Virginia University, Morgantown, WV 26506-6315, USA

Many important plasma processes, such as heating and current drive in tokamaks and laser-plasma interactions, involve the interaction of an externally generated electromagnetic wave with the plasma. The plasma can couple the incident wave to a range of other waves. Important examples include Raman scattering, where the incident wave couples to an electrostatic Langmuir wave and a scattered electromagnetic wave, and Brillouin scattering, where the Langmuir wave is replaced by an ion sound wave ${ }^{1}$. Both are observed in laser plasma experiments; however, these instabilities can also be generated by intense, short pulse microwave signals in a cool, tenuous plasma. A magnetized plasma supports additional beat wave interactions with natural cyclotron motions of charged particles and with magnetized plasma waves such as upper/lower hybrid waves as well as kinetic modes such as ion/ electron Bernstein waves. Applications include heating plasma that is inaccessible at low harmonics of the cyclotron frequency in high density fusion plasmas. Enabling these experiments is a large helicon apparatus, $1 \mathrm{~m}$ diameter, $3 \mathrm{~m}$ length. The stainless-steel vacuum vessel is surrounded by several electromagnets providing a $\mathrm{B}_{0}$ up to $0.0875 \mathrm{~T}$. The plasma will be ionized by $\mathrm{m}=0$ helicon waves with $3<\mathrm{f}<30 \mathrm{MHz}$ launched by a flat spiral antenna ${ }^{2}$. Plasmas of $10^{15}<n_{e}<10^{18}$ $\mathrm{m}^{-3}$ and $T_{e}<10 \mathrm{eV}$ will be produced in a low pressure $(<100$ mTorr) noble gas. R.F. compensated Langmuir probes and line integrated interferometry will be used to diagnose the plasma. These measurements will be carried out alongside numerical simulations and theoretical analysis to enhance the understanding of these interactions.

1. W. L. Kruer, 'The Physics of Laser Plasma Interactions', Westview Press, Boulder, Colorado, 2003

2. S. Shinohara et al., Phys. Plasmas, 16, 057104, 2009

Supported by UK EPSRC and US AFOSR. 